\title{
Test of the Anderson-Stuart model and correlation between free volume and the 'universal' conductivity in potassium silicate glasses
}

\author{
Marcio Luis Ferreira Nascimento ${ }^{a}, *$, Shigueo Watanabe ${ }^{b}$ \\ ${ }^{a}$ Vitreous Materials Laboratory, Department of Materials Engineering, Federal University of Sao Carlos, 13565-905 Sao Carlos-SP, Brazil \\ ${ }^{\mathrm{b}}$ Ionic Crystals, Thin Films \& Dating Laboratory, Institute of Physics, University of São Paulo, 05508-900 São Paulo-SP, Brazil
}

Received 22 March 2006; received in revised form 9 April 2007; accepted 29 April 2007

\begin{abstract}
Following recent finding it is shown that using conductivity and molar volume in binary potassium silicate glasses (considering the same batches) there is a common cubic scaling relation between them due to increase in alkali content. Emphasis is placed on the application of Anderson-Stuart model to describe the variation of activation enthalpy for conduction $E_{\mathrm{A}}$ with potassium concentration. In this analysis were considered experimental parameters, like shear modulus $G$ and relative dielectric permittivity $\varepsilon$, in wide composition range (between 1.7 and $40 \mathrm{~K}_{2} \mathrm{O}$ mol\%). The effects of $G, \varepsilon$ and free volume are taken into account. The drastic drop in conductivity up to 17 orders of magnitude for so many ion-conducting binary alkali silicate glasses is then mainly caused by the structure and the ion content. In particular, it is suggested that the glass network expansion, which is related to the available free volume, is a parameter that could explain the increase in ionic conductivity for this binary system.
\end{abstract}

(C) 2007 Elsevier B.V. All rights reserved.

PACS: 61.43.Fs; 66.10.Ed

Keywords: Ionic conductivity; Anderson-Stuart model; Free-volume

\section{Introduction}

High room temperature ionic conductivity in solid materials is technologically interesting for various solid-state electrochemical devices, such as batteries, sensors and 'smart windows'. It is well known that the ionic conductivity increases rapidly when a former glass network (for instance, $\mathrm{SiO}_{2}$ ) is modified by the addition of a metal alkali, such as $\mathrm{K}_{2} \mathrm{O}$. Despite considerable experimental and theoretical attempts, there is currently no consensus regarding the diffusion mechanism involved [1], even in simple systems. Several models have been proposed, and they range from thermodynamics models, with principles based on liquid electrolytes such as the weak electrolyte [2], to models based on solid-state concepts such as the jump diffusion [3], the strong electrolyte [4] and the microscopic dynamic structure [5].

Various models have been proposed for estimating the activation energy in alkali glasses. Particularly, a model suggested by Anderson and Stuart (A-S) [4] is considered to be the most

\footnotetext{
* Corresponding author. Tel.: +55 1633518556; fax: +55 1633615404.

E-mail address: pmlfn@iris.ufscar.br (M.L.F. Nascimento).
}

directly related to physically meaning parameters, such as ionic radii, relative dielectric permittivity and the elastic modulus. However, not many experimental data were available at the time the A-S theory was formulated. Moreover, no analysis has been performed for the potassium silicate system considering so many experimental data [4], and up to now only Hakim and Uhlmann [6] have proposed modifications on the A-S model, simply considering binary alkali silicate glasses.

The present paper reports data on the ionic conductivities and activation energies of glasses in the $\mathrm{K}_{2} \mathrm{O}-\mathrm{SiO}_{2}$ system, with the purpose of correlating new proposals to activation enthalpy with composition using experimental parameters data, such as shear modulus $G$ and relative dielectric permittivity $\varepsilon$. Also, a 'universal' finding is pursuit-using $\log _{10} \sigma$ versus $E_{\mathrm{A}} / k_{\mathrm{B}} T$. Selected experimental density data available were used to calculate the free volume in an attempt to evaluate proposals concerning the role of an open structure for ionic conductivity. We test a general relation between the ionic conductivity enhancement and the expansion of the network forming unities, which shows that the alkali-induced volume expansion of the glass network could partially explain ionic conductivity, and that is related to the shear modulus. Dielectric permittivity is also taken into account. 


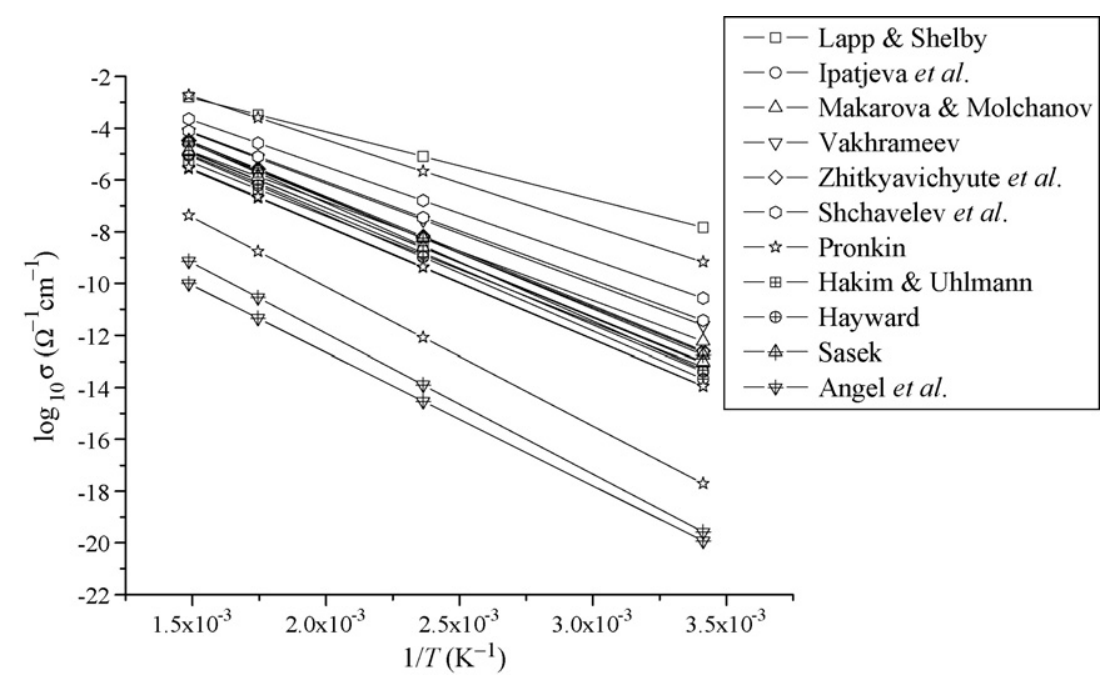

Fig. 1. Arrhenius plots of ionic conductivities in 21 binary potassium silicate glasses [7-21]. The temperatures measured are, respectively, $20,150,300$ and $400{ }^{\circ} \mathrm{C}$.

\section{Results and discussion}

Ionic conductivity $\sigma$ in glass is a thermally activated process of mobile ions that overcome a potential barrier $E_{\mathrm{A}}$, according to the following equation:

$\log _{10} \sigma=\log _{10} \sigma_{0}-\left(\log _{10} e\right) E_{\mathrm{A}} / k_{\mathrm{B}} T$

where $\sigma_{0}$ is the pre-exponential factor. In the following sections it will be shown that $\sigma_{0}$ does not depend on concentration or ion species. Fig. 1 presents experimental data on ionic conductivity of 21 binary potassium silicate glasses ranging from 20 up to $400^{\circ} \mathrm{C}$ that follows Eq. (1) [7-21]. As will be detailed below, such equation should be more usefull when one considers $\sigma=\sigma\left(E_{\mathrm{A}}, T\right)$, leading, in fact, to a more general rule.

Many experimental works on conductivity in potassium silicate glasses have been published over years, basically searching for the highest conductivity values or presenting theories that apply better in certain systems, as will be cited below. However, in many works one could observe that parameters such as kind of electrode and its influence, surface preparation and conditions, heat treatments, phases involved, and others have been omitted. For common glasses at room temperatures, the conductivities as low as $10^{-17} \Omega^{-1} \mathrm{~cm}^{-1}$, approaching the limit of the available measuring apparatus. At high alkali content, the samples are hygroscopic, and special care on preparation procedures must be exercised.

Differences observed in the activation enthalpies, shown in Fig. 1 are likely to be associated with differences in the chemistry and/or structure of the glass samples. Fig. 2 confirms this fact showing experimental density data $(d)$ for the same batches presented in Fig. 1 [7-21]. An increase in $d$ (or a decrease in $V_{\mathrm{m}}$ ) with alkali content can be noticed. As the structure becomes more compact with increasing alkali content, the conductivity increases. A simple question arises: how do potassium ions move in this system? Following are proposed explanations for this question.

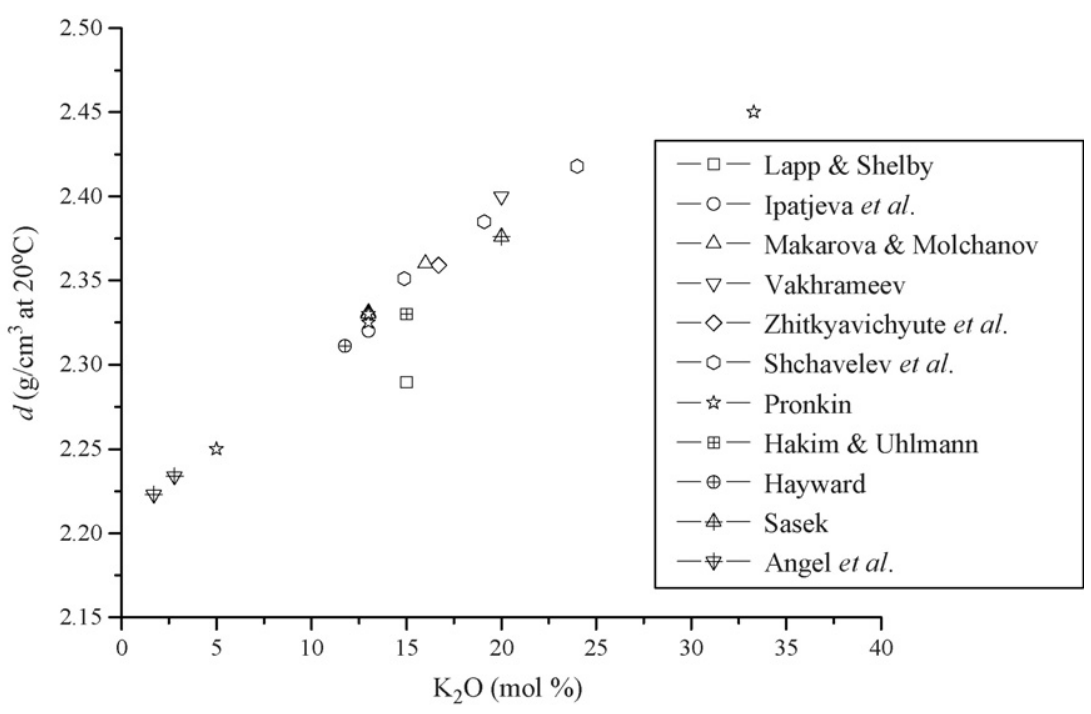

Fig. 2. Densities measured at $20^{\circ} \mathrm{C}$ of 21 binary potassium silicate glasses (the same glasses presented in Fig. 1 [7-21]). 
Also, differences in both melting and annealing procedures, sample preparation techniques and water content could affect the conduction results. In the $\mathrm{K}_{2} \mathrm{O}-\mathrm{SiO}_{2}$ composition system, further differences could arise from the discussion on effects of phase separation [22].

\subsection{The Anderson-Stuart model}

Despite differences in the activation energies observed by different investigators, a number of common trends can be seen in these data. Perhaps most important is the decrease in activation enthalpy with increasing the potassium concentration. It is interesting, therefore, to evaluate whether or not such behaviour can be predicted from a model proposed by Anderson and Stuart [4]. In this 'classical' model, the activation enthalpy for conductivity may be divided in two parts: the electrostatic binding energy of the original site, $E_{\mathrm{b}}$, and the strain energy, $E_{\mathrm{s}}$, required to move an ion from one site to another. $E_{\mathrm{b}}$ describes the coulombic forces acting on the ion as it moves away from its charge-compensating site, and $E_{\mathrm{s}}$ describes the mechanical forces acting on the ion as it dilates sufficiently the structure to allow the ion to move between sites. The basic idea is that an ion (in this case $\mathrm{K}^{+}$) makes a simple jump from one site to another, and passes through a 'doorway' which opens as it passes through, where cations sites require only the presence of non-bridging oxygens. The A-S model follows Eq. (2):

$E_{\mathrm{A}}=\frac{\beta z z_{0} e^{2}}{\gamma\left(r_{\mathrm{K}}+r_{\mathrm{O}}\right)}+4 \pi G r_{\mathrm{D}}\left(r_{\mathrm{K}}-r_{\mathrm{D}}\right)^{2}$

where $z$ and $z_{0}$ are the valences of the mobile ion and of the fixed counterion-in this case potassium and oxygen, respectively, $r_{\mathrm{K}}$ and $r_{\mathrm{O}}$ the corresponding Pauling ionic radii for $\mathrm{K}^{+}$ and $\mathrm{O}^{2-}, e$ the electronic charge and $r_{\mathrm{D}}$ is the effective radius of the (unopened) doorway.

The parameters of interest in the A-S model are the elastic modulus $(G)$, the 'Madelung' constant $(\beta)$, which depends on how far apart the ions are and a covalency parameter $(\gamma)$, which indicates the degree of charge neutralization between the ion and its nearest neighbours. In their original paper [4], Anderson and Stuart assumed that this covalency parameter could be taken equal to a typical value of the relative dielectric permittivity $(\varepsilon)$. Martin and Angell [23] have provided a visualization of the energetics of the conduction process in an ionic conducting glass based on the A-S model. McElfresh and Howitt [24] suggested a modification on the $E_{\mathrm{S}}$ term, where they included the jumping distance $\lambda$ as a more appropriated parameter (Eq. (3)).

$E_{\mathrm{A}}=\frac{\beta z z_{0} e^{2}}{\gamma\left(r_{\mathrm{K}}+r_{\mathrm{O}}\right)}+4 \pi G \lambda\left(r_{\mathrm{K}}-r_{\mathrm{D}}\right)^{2}$

Two considerations are proposed here: (a) one is related with the shear modulus $G$. Besides scattering, available $G$ data increase slightly with increasing $\mathrm{K}_{2} \mathrm{O}$ mol\% concentration, as shown in Fig. 3; (b) following A-S theory, we considered the experimental relative dielectric permittivity $\varepsilon$ [4]. We also con-

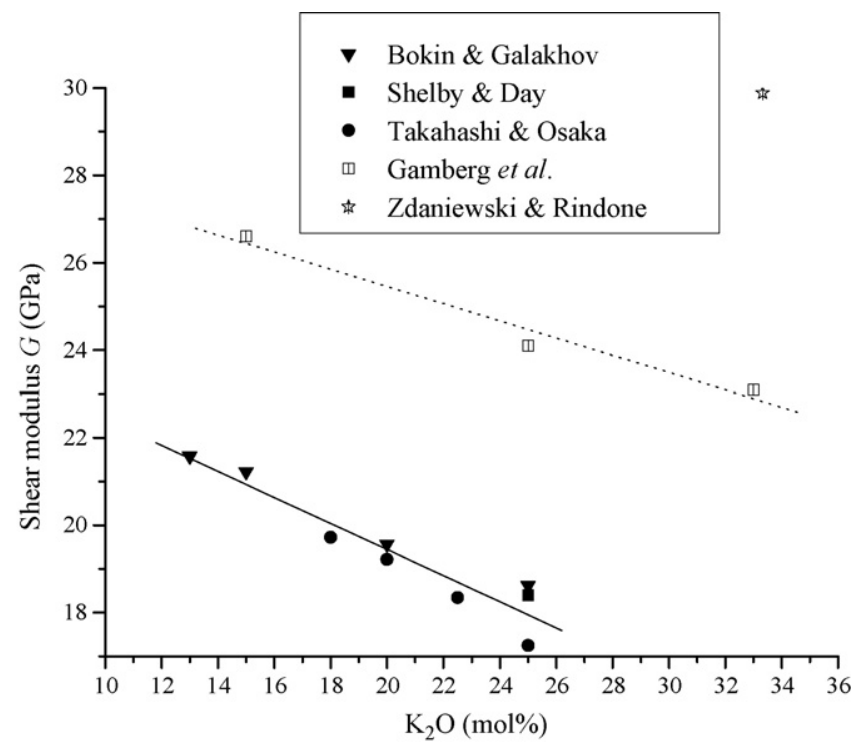

Fig. 3. Measured shear modulus ( $G$, in $\mathrm{GPa}$ ) of $\mathrm{K}_{2} \mathrm{O}-\mathrm{SiO}_{2}$ system [25-29]. Data from Bokin and Galakhov, Shelby and Day, and Takahashi and Osaka were considered in the A-S model, but not Gamberg et al.'s and Zdaniewski and Rindone's data.

sider $\beta$ as in the Anderson and Stuart model [4]:

$\beta=\frac{a-r_{\mathrm{K}}}{b}$

where $r_{\mathrm{K}}$ is the value given in $\AA$, also with $a$ and $b$, that will be defined below.

Two hypotheses were taken into account in this work: (i) $r_{\mathrm{D}}$ fitting all data, as suggested by the A-S theory; (ii) $\lambda$ fitting all data, following McElfresh and Howitt's suggestion [24], Eq. (3). In this case $r_{\mathrm{D}}$ was considered as a fitting parameter, just for comparison.

From Fig. 3, the shear modulus $G$ from Bokin and Galakhov, Takahashi and Osaka, and Shelby and Day [25,26,28] showed a decrease with increasing potassium content. Data from Gamberg et al. [27] also demonstrated a decrease with increasing $\mathrm{K}_{2} \mathrm{O}$ content, but they measured $G$ at $3 \mathrm{kbar}$ pressure. Zdaniewski and Rindone [29] measured $G$ under $\mathrm{H}_{2} \mathrm{O}$ atmosphere. So, these last two data sets were not considered. In this work we followed a linear fit (Eq. (5)):

$G=G_{0}-\frac{\mathrm{d} G}{\mathrm{~d} n} n$,

where $G_{0}=(25.41 \pm 0.77) \mathrm{GPa}, n$ the $\mathrm{K}_{2} \mathrm{O}$ mol\% composition and $\mathrm{d} G / \mathrm{d} n=(0.298 \pm 0.037) \mathrm{GPa} m o l \%^{-1}$, with correlation factor $R^{2}=0.95$. It is recognized that this assumption of a similitude of form may provide an inadequate description of the change in $G$ with alkali concentration; therefore it seems to provide only an approximation.

In Fig. 4, the relative dielectric permittivity $\varepsilon$ from many authors [30-33] showed a small and monotonic increase with increasing potassium content, according to Eq. (6):

$\varepsilon=\varepsilon_{0}+\frac{\mathrm{d} \varepsilon}{\mathrm{d} n} n$, 


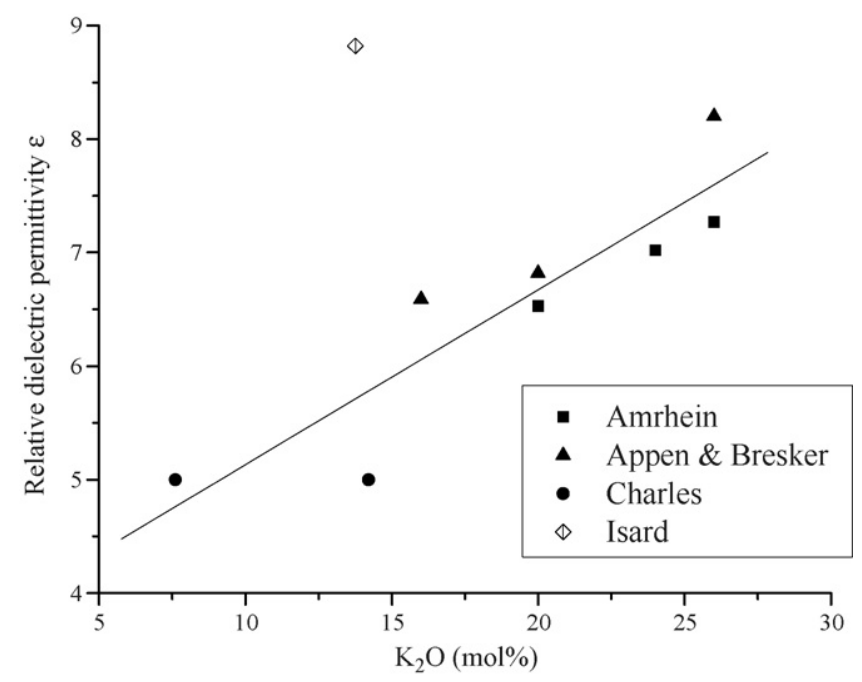

Fig. 4. Experimental relative dielectric permittivity values $(\varepsilon)$ of $\mathrm{K}_{2} \mathrm{O}-\mathrm{SiO}_{2}$ system [30-33]. Only data from Isard [32] were discarded from analysis.

where $\quad R^{2}=0.90, \quad \varepsilon_{0}=3.59 \pm 0.60 \quad$ and $\quad \mathrm{d} \varepsilon / \mathrm{d} n=(0.154 \pm$ 0.030) $\mathrm{mol}^{-1}$, just considering Amrhein, Appen and Bresker and Charles' data. Only data from Isard [32] was discarded from analysis.

The variation of activation enthalpy for conduction $E_{\mathrm{A}}$ with $\mathrm{K}_{2} \mathrm{O}$ mol\% content over 21 glasses is shown in Fig. 5, and these data correspond to the same experimental data in Figs. 1 and 2.

A carefull analysis was carried out to find some possible discrepancies on the scattering. For example, phase separation has also been shown to play a major role in controlling the conductivity properties of glasses. In many cases the morphology of the glass is far more important than the bulk composition in determining this property [33]. Ionic diffusion involves mass transport over extended distances, and is particularly sensitive to changes in morphology. It follows that both bulk composition and thermal history play important roles in controlling the properties of phase-separated glasses. The existence of phase separation in potassium silicate glasses was discussed as long ago as 1965 by Charles [33], but little attention has been paid to this problem since that time. Unfortunately, the potassium silicate system showed some slight experimental evidence of structural segregation [33,34]. According to theoretical work [22] the immiscibility range of potassium silicate glasses is estimated to be between near pure silica and $15 \mathrm{~K}_{2} \mathrm{O}$ mol\% addition.

At high alkali content, the samples are hygroscopic, and special precautions in the preparation and measuring procedures must be taken to ensure the absence of surface or bulk proton conduction (from water content). These processes would lead to higher $\sigma$ conductivities, and consequently to lower $E_{\mathrm{A}}$ activation enthalpies. No water content effect on conduction in potassium silicate glass has been performed from all authors cited, but in the $\mathrm{Na}_{2} \mathrm{O} \cdot 3 \mathrm{SiO}_{2}$ system Takata et al. [35] observed that the conductivity-water content relation is similar to that observed in the "mixed-alkali" effect, and suggested a similar interaction between $\mathrm{Na}^{+}$and water $\left(\mathrm{H}^{+}\right.$or $\left.\mathrm{H}_{3} \mathrm{O}^{+}\right)$.

Besides some scattering, effects of glass composition on $E_{\mathrm{A}}$ could be parametrized by the A-S theory. This model could even be applied in alkali silicate glasses to predict, for example, the dependence of $E_{\mathrm{A}}$ with $\mathrm{K}_{2} \mathrm{O}$ content considering non-separated glassy samples. Fig. 5 presents data that support the discussion above.

With regard to the fitting procedure, in the first case radii values were fixed as in the $\mathrm{A}-\mathrm{S}$ model for the potassium silicate system $\left(r_{\mathrm{K}}=1.33 \AA\right.$ and $r_{\mathrm{O}}=1.4 \AA$, full line $)$. The fitting parameter was the doorway radius, that resulted in $r_{\mathrm{D}}=0.92 \AA$. The second assumption was a high 'doorway' radius value giving $r_{\mathrm{D}}=0.93 \AA$ and a jumping distance $\lambda=1.7 \AA$, and both were used as fitting parameters. Shear modulus $G$ and the relative dielectric permittivity $\varepsilon$ were used on both assumptions for all data (Figs. 3 and 4 ). The $\beta$ parameter used resulted in 0.23 considering assumptions $i$ and $i i(a=2.05 ; b=3.20)$ respectively. Just for comparison, the $\beta$ value for the potassium silicate system was also 0.33 , and it is possible to presume that the values presented are consistent.

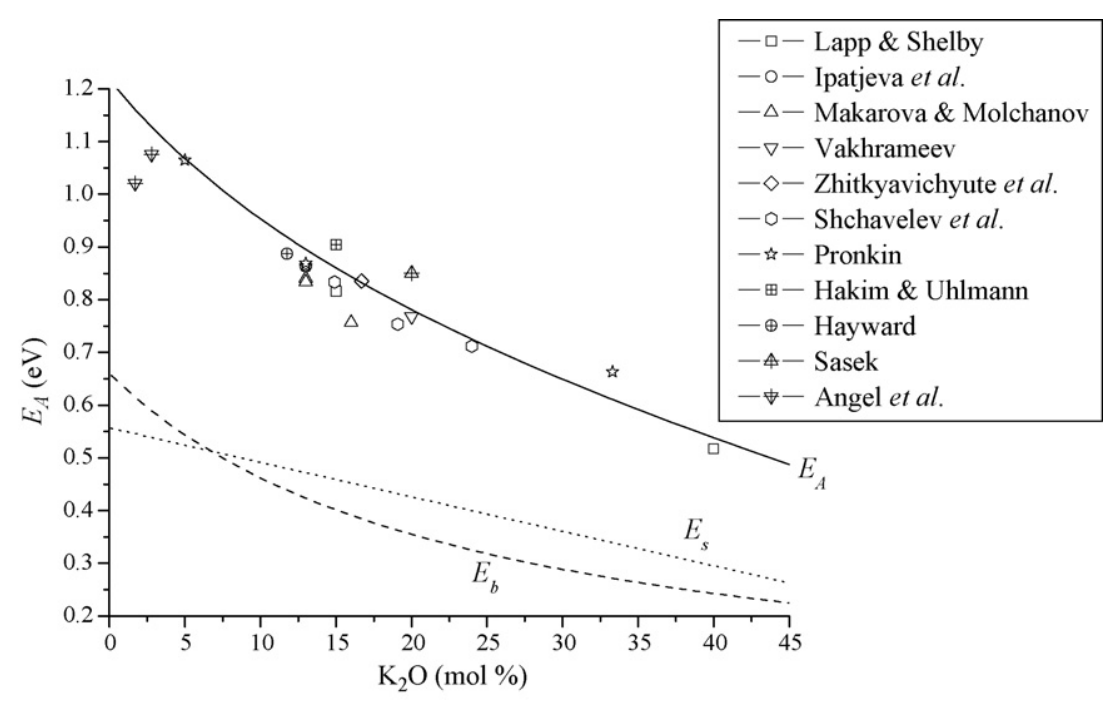

Fig. 5. Non-linear adjustment (full line) on activation enthalpies in $21 \mathrm{~K}_{2} \mathrm{O}-\mathrm{SiO}_{2}$ glasses [7-21] considering assumption $i i$. $E_{\mathrm{b}}$ is the binding energy (dashed line), and $E_{\mathrm{S}}$ the strain energy (dotted line) terms. 
The adjustment for activation enthalpy $E_{\mathrm{A}}$ in Fig. 5 was performed using a Levenberg-Marquardt non-linear fitting. It is surprising that a simple theory could adjust a lot of data from several authors with different glass preparation processes in a wide composition range. With respect to the model, the analysis using shear modulus $G$ showed more influence on the A-S theory than using experimental relative dielectric permittivity $\varepsilon$, considering hypothesis $i$.

In summary, results in Fig. 5 showed that $E_{\mathrm{s}}$ is higher than $E_{\mathrm{b}}$ considering the Anderson-Stuart [4] hypothesis (i). The McElfresh and Howitt [24] hypothesis (ii) presented similar results (i.e., $E_{\mathrm{b}}+E_{\mathrm{s}}$ ). It is interesting to note that the $E_{\mathrm{b}}$ dependence on the $\mathrm{K}_{2} \mathrm{O}$ content is related to the relative dieletric permittivity $\varepsilon$, which increases with the potassium content. The major difference between hypotheses (i) and (ii) is firstly related to the experimental shear modulus $G$. Thus, the doorway radius $r_{D}$ and the jump distance $\lambda$ must have only strong influence on glass systems that present major influence on $E_{\mathrm{s}}$.

\subsection{The 'universal' conductivity and the free volume}

Extensive studies have recently been made for obtaining a 'universal' equation (or "master curve") from the glass structure standpoint. For example, Nascimento et al. [36] presented 23 and 30 binary rubidium and cesium silicate glasses, respectively, that follows a 'universal' conductivity rule. Swenson and Börjesson [37] proposed a common cubic scaling relation between $\sigma$ and the expansion volumes of the networking forming units in salt-doped and -undoped glasses. This fact suggested that the glass network expansion, which is related to the available free volume, is a key parameter determining the increase of the high ionic conductivity in some types of fast ion-conducting glasses.

According to Adams and Swenson [38], the ion conduction should be determined by the ionic motion within an infinite pathway cluster. For various silver ion-conducting glasses [39,40], it was found that the cubic root of the volume fraction $F$ of infinite pathways for a fixed valence mismatch threshold is closely related to both the absolute conductivity and the activation enthalpy of the conduction process:

$\log _{10} \sigma T \approx \sqrt[3]{F}=\log _{10} \sigma_{0}^{\prime}-\left(\log _{10} e\right) E_{\mathrm{A}} / k_{\mathrm{B}} T$

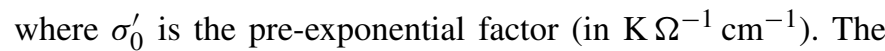
cubic root of $F$ may be thought of as proportional to the mean free path length of the mobile ion [41].

Nascimento and Watanabe [42-46] have recently verified this 'universal' finding in binary silicate, borate and germanate glasses, considering both Eqs. (1) and (7). This paper aimed to present new results considering just potassium silicate glasses. From these studies, the influence of alkali content and temperature was minor on the pre-exponential terms, considering both expressions $\log _{10} \sigma$ and $\log _{10} \sigma T$.

Fig. 6 shows modified Arrhenius plots of $\sigma$ for the 21 potassium silicate glasses (from $x=1.7$ to $40 \mathrm{~mol} \%$, as indicated), ranging from $1.5 \times 10^{-3}$ to $1.2 \times 10^{-20} \Omega^{-1} \mathrm{~cm}^{-1}$ between 20 and $400^{\circ} \mathrm{C}$. The range of activation enthalpy $E_{\mathrm{A}}$ lies between 0.52 and $1.07 \mathrm{eV}$ in all glasses studied, as indicated in Fig. 5. These data were compared with the 'universal' equation for $\sigma_{0}=50 \Omega^{-1} \mathrm{~cm}^{-1}$ in Eq. (1). Following previous work by Nascimento et al., this "universal" equation appears in Fig. 6 as a dashed line, and the dotted lines are the higher and lower limits within one magnitude order. Only glasses with few $\mathrm{K}_{2} \mathrm{O}$ mol\% does not obey the 'universal' finding, as Angel et al.'s data.

The replacement of a mobile ion with one of another type affects the ionic conductivity in various ways, such as causing modifications in the glass structure. But considering so many different binary potassium silicate glasses, as in Fig. 6, it is remarkable how strong is the correlation between $\sigma$ with $E_{\mathrm{A}}$ and $T$.

The fact is that $\sigma$ lies on a single 'universal' curve in various potassium silicate glasses, whose conductivities differ by more than 17 orders of magnitude and within scatters of just one

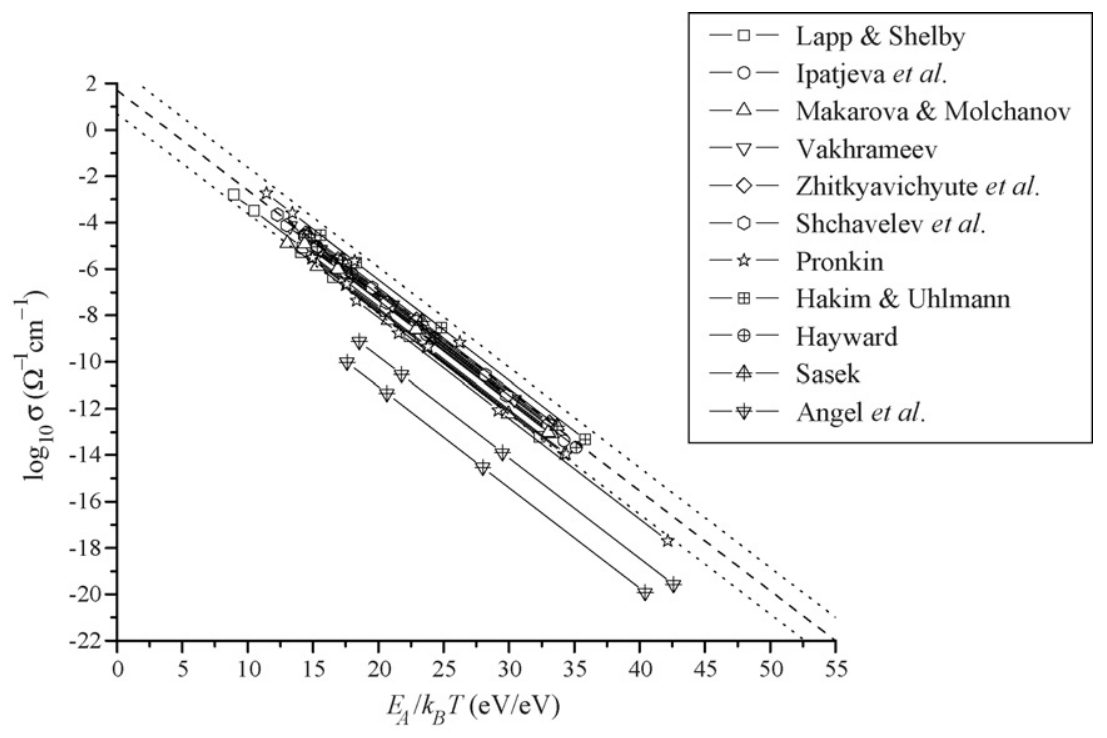

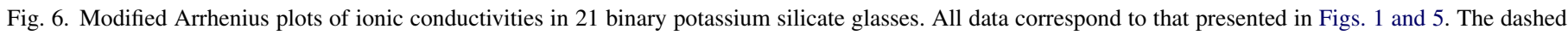
line is the 'universal curve', Eq. (1), with $\sigma_{0}=50 \Omega^{-1} \mathrm{~cm}^{-1}$, and the dotted lines correspond to one order of magnitude higher or lower than Eq. (1). 


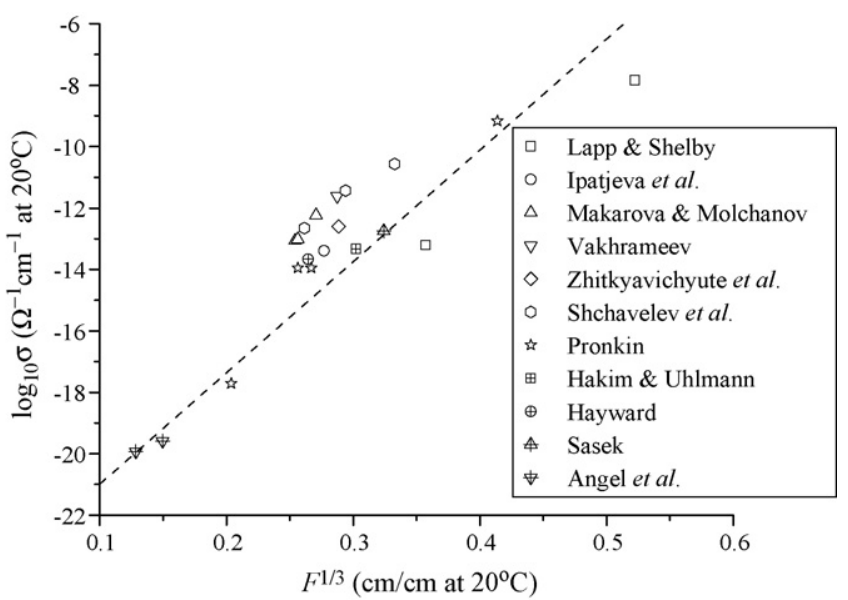

Fig. 7. Measured ionic conductivity $\sigma$ at $20^{\circ} \mathrm{C}$ vs. the cubic root of expansion of glass network $F$ estimated from density measurements at same temperature.

order of magnitude in $90 \%$ of the glass systems considered. It is also important to notice the wide composition range involved. Therefore, if one measures $\sigma$ at a fixed temperature, it is possible to estimate $E_{\mathrm{A}}$ from Eq. (1) considering $\sigma_{0}=50 \Omega^{-1} \mathrm{~cm}^{-1}$, and obtain a rough sketch of $\sigma$ at different temperatures. This means that, if $E_{\mathrm{A}}$ is obtained by some experimental or theoretical technique, the ionic conductivity can be readily calculated.

Another "universal" curve, following Eq. (7) and considering some binary silicate, borate and germanate glasses, resulted in the same 'universal' behaviour [42-46], as cited above. The pre-exponential value was $\sigma_{0}^{\prime}=50,000 \mathrm{~K}^{-1} \mathrm{~cm}^{-1}$. The conclusions for this case also follow the above described considering Eq. (1). The most important fact is that in Fig. 6 data for glasses of different compositions are unified by the single 'universal' Eq. (1). The fact that $\sigma$ lies on this single 'universal' curve for many ion-conducting glasses means that $\sigma$ is mainly governed by $E_{\mathrm{A}}$.
Therefore, Fig. 6 shows a 'universal curve' plotting conductivities and activation enthalpies. In order to investigate the possibility of another general relation between ionic conductivity and the volume occupied by the network skeleton, the authors have calculated the expansion $\left(V_{\mathrm{g}}-V_{\mathrm{m}}\right) / V_{\mathrm{m}}$ of the network, where $V_{\mathrm{m}}$ and $V_{\mathrm{g}}$ are the volumes of the network forming unities $\left(\mathrm{SiO}_{2}\right)$ occupy in the doped $\left(\mathrm{K}_{2} \mathrm{O}-\mathrm{SiO}_{2}\right)$ glasses, respectively. As shown in Fig. 2, the dopant $\mathrm{K}_{2} \mathrm{O}$ added increases the experimental density before occupied by $\mathrm{SiO}_{2}$, where density of pure silica was assumed as $2.2064 \mathrm{~g} \mathrm{~cm}^{-3}$. It is important to note that conductivity and density (or molar volume) data were considered from the same batches in Figs. 1 and 2, respectively.

The glass systems in Fig. 7 cover a wide composition range, between 1.7 and $40 \mathrm{~mol} \%$. A striking common relation between the conductivity at $20^{\circ} \mathrm{C}$ and the cubic root of free volume $F=\left(V_{\mathrm{g}}-V_{\mathrm{m}}\right) / V_{\mathrm{m}}$ calculated from density of the same batches and at same temperature is evident; i.e., for a given expansion all the different systems respond with the same increase of $\sigma$, regardless of chemical (such as relative water content) or microstructural details (such as phase separation). This is a rather rough approximation: the increase in molar volume of $\mathrm{K}_{2} \mathrm{O}$ unities is the main factor involved in the increasing in conductivity and also in the free volume. Thus, the free volume defined here is a macroscopic quantity. The necessary condition for ion transport may rather be the presence of microscopic pathways available for potassium ions. A given material may be called 'conductive' if it is equipped with ample ionic pathways, irrespective of the amount of the free volume.

Note that the data in Fig. 7 represent conductivity values that vary by 13 orders of magnitude. The relation found is approximately linear, what could mean in a first approximation that the conductivity (or $\sigma T$ ) is less dependent on the number of mobile ions than the on free volume itself.

The common behaviour of the conductivity increase with expansion of the network structure observed for the various

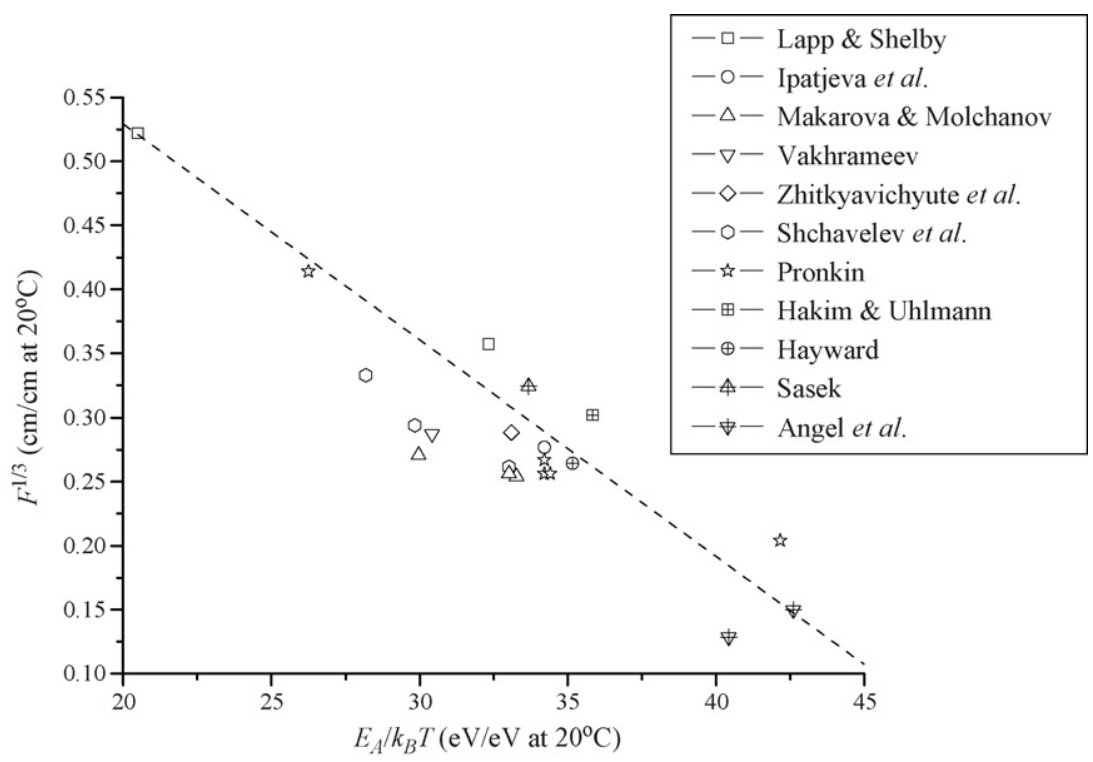

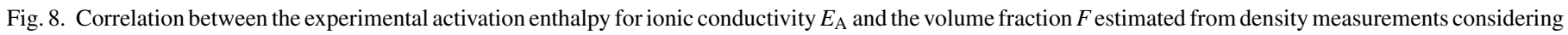
a fixed temperature of $20^{\circ} \mathrm{C}$. 
binary potassium glasses suggests that the excess volume introduced by the dopant is a key parameter that determines the conductivity properties, as expressed by Eq. (7). Thus, at first sight it appears that the details of the microscopic structure have direct impact on the ionic conductivity in this system. However, it should be noted that the microscopic interactions (mainly mechanical and dielectrical, as predicted by Anderson-Stuart theory) lead to variations of the degree of expansion. For this reason, in order to explain the conducting properties and the increase the ionic conductivity with alkali content we focused on the A-S theory.

It is interesting to note that the increase in ionic conductivity is almost entirely due to the fact that the activation enthalpy $E_{\mathrm{A}}$ required for a cation jump decreases, as presented in Fig. 5. Thus, the pre-exponential term in the Arrhenius law in Eq. (1), $\sigma_{0}$, is largely unaffected by alkali addition, as showed in Fig. 6 .

The present finding of the common scaling between the conductivity enhancement and the expansion suggests that it is the expansion of the glass skeleton and therefore the strain energy part $E_{\mathrm{s}}$ influence on the conduction properties in this system, as presented in Fig. 6.

Fig. 8 shows that an increase in volume fraction reduces the activation enthalpy for an ionic jump, which demonstrates that $E_{\mathrm{A}} / k_{\mathrm{B}} T$ varies linearly with the cube root of the volume fraction $F$. Thus, this approach emphasizes the importance of "free volume" to the ion mobility.

\section{Conclusions}

The Anderson-Stuart model (A-S) has been used for a potassium-silica glass system to describe the variation of the activation enthalpy against composition in a wide composition range (from 0.1 up to $45 \mathrm{~K}_{2} \mathrm{O}$ mol\%), with reasonable results. Theoretical activation enthalpy $E_{\mathrm{A}}$ of potassium silicate glasses seems to vary smoothly with composition. For the first time the considerations on experimental shear modulus $G$ and relative dielectric permittivity $\varepsilon$ with potassium composition fit well experimental available data. Shear stress has given more influence on fitting than relative dielectric permittivity. Considering the A-S theory, potassium silicate conducting glasses fall into an identifiable pattern where conductivity is related to chemical composition. A 'universal' finding is obtained using $\log _{10} \sigma$ versus $E_{\mathrm{A}} / k_{\mathrm{B}} T$ in this binary silicate system. As a consequence, the pre-exponential factor of the conductivity $\sigma_{0}$ is composition independent. Another same and simple relation between the increase in ionic conductivity and the expansion of the glass network skeleton is revealed for very different binary potassium silicate glasses. The results show that an open structure with excess free volume, in addition to the alkali content, plays a significant role in promoting ionic conductivity. One should point that that the true free volume (from theory) is limited in all glasses, irrespective of the network modifier or dopant concentration. Therefore, the approach considered here was to relate ionic conductivity with the "expansion of the glass network" (or the "free volume") originated from the conduction pathways.

\section{Acknowledgement}

This work was financially supported by the Brazilian agency FAPESP (grant no. 04/10703-0).

\section{References}

[1] C.A. Angell, Ann. Rev. Phys. Chem. 43 (1992) 693.

[2] D. Ravaine, J.L. Souquet, Phys. Chem. Glasses 18 (1977) 27.

[3] K. Funke, Prog. Solid State Chem. 22 (1993) 11.

[4] O.L. Anderson, D.A. Stuart, J. Am. Ceram. Soc. 37 (1954) 573.

[5] A. Bunde, M.D. Ingram, P. Maass, J. Non-Cryst. Solids 172/174 (1994) 1222.

[6] R.M. Hakim, D.R. Uhlmann, Phys. Chem. Glasses 12 (1971) 132.

[7] J.C. Lapp, J.E. Shelby, J. Non-Cryst. Solids 84 (1986) 463.

[8] J.C. Lapp, J.E. Shelby, J. Non-Cryst. Solids 86 (1986) 350.

[9] V.V. Ipatjeva, Z.U. Borisova, V.S. Molchanov, Zh. Prikl. Khim. 40 (1967) 1424.

[10] T.M. Makarova, V.S. Molchanov, Opt. Mekh. Prom. 2 (1961) 26.

[11] T.M. Makarova, O.V. Mazurin, V.S. Molchanov, Izv. Vyssh. Uchebn. Zaved., Khimiya i Khim. Tekhnol. 3 (1960) 1072.

[12] V.I. Vakhrameev, Steklo 3 (1968) 84.

[13] I.I. Zhitkyavichyute, V.S. Molchanov, F.K. Aleinikov, Tr. Akad. Nauk Litov. SSR, B 2 (1965) 137.

[14] O.S. Shchavelev, S.S. Kasymova, R.V. Sipovskaya, Opt. Mekh. Prom. 5 (1973) 40.

[15] A.A. Pronkin, Khimiya Tverdogo Tela, Leningrad Gos. Univ., Leningrad, 1965, p. 125.

[16] A.A. Pronkin, Fizika i Khimiya Stekla 5 (1979) 634

[17] A.A. Pronkin, Fizika i Khimiya Stekla 6 (1980) 742.

[18] R.M. Hakim, D.R. Uhlmann, Phys. Chem. Glasses 8 (1967) 174.

[19] P.J. Hayward, Phys. Chem. Glasses 17 (1976) 54.

[20] L. Sasek, Sb. Vys. Sk. Chem. Technol. Praze, Chem. Technol. Silik. L6 (1975) 61.

[21] P.W. Angel, R.E. Hann, A.R. Cooper, J. Non-Cryst. Solids 183 (1995) 277.

[22] C. Huang, A.N. Cormack, J. Chem. Phys. 95 (1991) 3634.

[23] S.W. Martin, C.A. Angell, J. Non-Cryst. Solids 83 (1986) 185.

[24] D.K. McElfresh, D.G. Howitt, J. Am. Ceram. Soc. 69 (1986) C237.

[25] P.Ya. Bokin, F.Ya. Galakhov, Neorg. Mater. 6 (1970) 2027.

[26] K. Takahashi, A. Osaka, J. Ceram. Soc. Jpn. 91 (1983) 116.

[27] E. Gamberg, D.R. Uhlmann, D.H. Chung, J. Non-Cryst. Solids 13 (1973) 399.

[28] J.E. Shelby, D.E. Day, J. Am. Ceram. Soc. 53 (1970) 182.

[29] W.A. Zdaniewski, G.E. Rindone, J. Am. Ceram. Soc. 64 (1981) C183.

[30] E.M. Amrhein, Glastech. Ber. 36 (1963) 425.

[31] A.A. Appen, R.I. Bresker, Zh. Tekhn. Fiz. 22 (1952) 946.

[32] J.O. Isard, Ph.D. Thesis, Sheffield, 1962.

[33] R.J. Charles, J. Am. Ceram. Soc. 49 (1966) 55

[34] Y.P. Gupta, U.D. Mishra, J. Phys. Chem. Solids 30 (1969) 1327.

[35] M. Takata, M. Tomozawa, E.B. Watson, J. Am. Ceram. Soc. 65 (1982) 91.

[36] M.L.F. Nascimento, E. do Nascimento, S. Watanabe, Braz. J. Phys. 35 (2005) 626.

[37] J. Swenson, L. Börjesson, Phys. Rev. Lett. 77 (1996) 3569.

[38] S. Adams, J. Swenson, Phys. Chem. Chem. Phys. 4 (2002) 3179.

[39] J. Swenson, S. Adams, Phys. Rev. B 64 (2002) 024204.

[40] S. Adams, J. Swenson, Solid State Ionics 154/155 (2002) 151.

[41] S. Adams, J. Swenson, Phys. Rev. Lett. 84 (2000) 4144.

[42] M.L.F. Nascimento, S. Watanabe, J. Mater. Sci. 40 (2005) 5079-5081.

[43] M.L.F. Nascimento, S. Watanabe, J. Mater. Sci. 40 (2005) 4423-4425.

[44] M.L.F. Nascimento, E. do Nascimento, S. Watanabe, Mater. Chem. Phys. 96 (2006) 55-58.

[45] M.L.F. Nascimento, S. Watanabe, Braz. J. Phys. 36 (2006) 795-798.

[46] M.L.F. Nascimento, E. do Nascimento, W.M. Pontuschka, M. Matusoka, S. Watanabe, Ceramica 52 (2006) 22-30. 